\title{
Feedback Control of Flight Speed to Reduce Unmanned Aerial System Noise
}

\author{
Matthew B. Galles, ${ }^{*}$ Noah H. Schiller ${ }^{\dagger}$ and Kasey A. Ackerman ${ }^{\ddagger}$ \\ NASA Langley Research Center, Hampton, Virginia 23681 \\ Brett Newman ${ }^{\S}$ \\ Old Dominion University, Norfolk, Virginia 23529
}

\begin{abstract}
The aim of this initial study was to incorporate an acoustic metric into the flight control system of an unmanned aerial vehicle. This could be used to mitigate the noise impact of unmanned aerial systems operating near residential communities. To incorporate an acoustic metric into a flight control system, two things were required: a source noise model, and an acoustic controller. An acoustic model was developed based on Gutin's work to estimate propeller noise. The flight control system was augmented with a controller to reduce propeller noise using feedback control of the commanded flight speed until an acoustic target was met. This control approach focused on modifying flight speed only, with no perturbation to the trajectory. Multiple flight simulations were performed and the results show that integrating an acoustic metric into the flight control system of an unmanned aerial system is possible.
\end{abstract}

\section{Nomenclature}

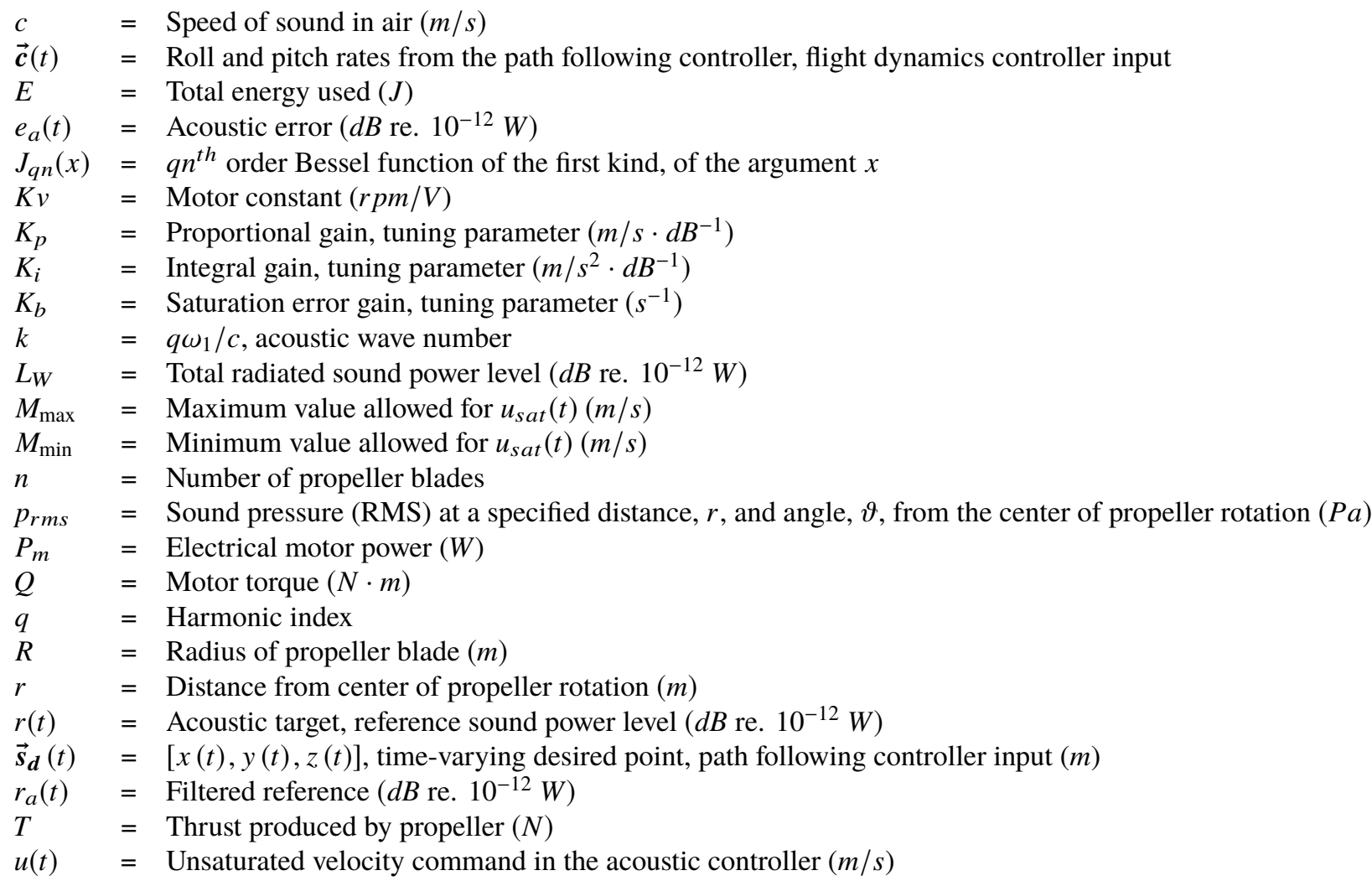

\footnotetext{
*Pathways Intern, Structural Acoustics Branch, MS 463, Hampton, VA 23681, AIAA Student Member.

${ }^{\dagger}$ Research Engineer, Structural Acoustics Branch, MS 463, Hampton, VA 23681, AIAA Member.

† Pathways Intern, Dynamic Systems and Control Branch, MS 308, Hampton, VA 23681, AIAA Student Member.

${ }^{\S}$ Professor, Dept. of Mechanical \& Aerospace Engineering, Old Dominion Univ., Norfolk, VA 23529, AIAA Associate Fellow.
} 


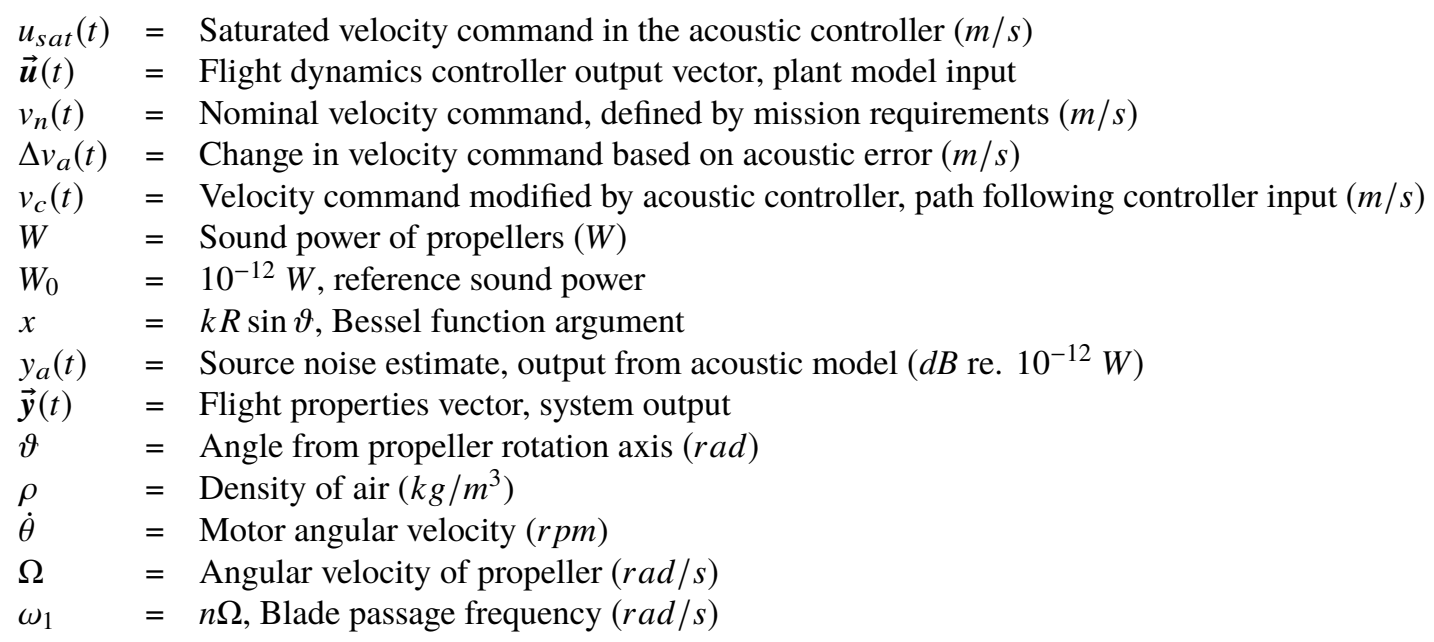

\section{Introduction}

D OPULARITY of Unmanned Aerial Systems (UAS) is on the rise, and as a result, flight noise could become a major barrier to public acceptance. Therefore, noise control technologies are necessary to mitigate noise impact during operations. The development of an acoustically-aware vehicle, which has the ability to modify where and how the aircraft flies to meet a noise requirement, as well as other mission requirements, could reduce noise exposure and enable widespread use of these vehicles in close proximity to people.

The objective of this paper is to demonstrate the feasibility of incorporating an acoustic target into the flight control system of a UAS as a first step toward an acoustically-aware vehicle. Flight speed is modified to satisfy a time-varying constraint on sound generated by the vehicle, or acoustic target value. Depicted in Fig. 1 is a simplified representation of the control architecture used in this study. Specifically, a feedback control system is implemented to modify a nominal velocity command, $v_{n}(t)$, to the vehicle system based on an acoustic reference, $r(t)$, and a noise metric value, $y_{a}(t)$. The acoustic reference value is specified a priori, and the noise metric value for the vehicle is estimated using a source noise model. As the figure shows, this approach requires at least two components: a source noise model and a control-law to incorporate the noise metric into the flight control system. Both components will be discussed in the next several sections along with the vehicle system, which includes the flight control system and vehicle dynamics model.

Section II provides a background of the vehicle system, baseline flight control system, and energy model. Section III covers the development of a source noise model for the vehicle. Section IV describes the control approach used to integrate an acoustics metric into the flight control system. Results of this study are presented in Section V.

\section{Vehicle System}

A vehicle system model is necessary to perform the feasibility study. This section describes the system models embedded in the vehicle system block shown in Fig. 1, including an energy consumption model that will be used to evaluate the effect of acoustic control on the operating efficiency of the aircraft.

\section{A. Vehicle System Model}

The vehicle system model used in this work is based on the GL-10 [1, 2], a 10-propeller distributed electric propulsion prototype aircraft, depicted in both hover and cruise flight configurations in Fig. 2. A vehicle system model that was previously created for GL-10 flight controls development will be used in this study; only the cruise flight configuration is considered in this work.

The vehicle system consists of four components, shown in Fig. 3. The first three components comprise the closed-loop vehicle model, consisting of a path following control-law, a flight dynamics controller, and a vehicle dynamics model. The path following controller implements the approach proposed by Cichella et al. [3, 4], which ensures a singularity-free control-law. The flight dynamics controller is a rate-tracking autopilot designed using classical control design techniques and uses a Proportional-Integral (PI) rate-tracking control-law with an $\mathcal{L}_{1}$ adaptive augmentation strategy [5-7] to compensate for disturbances and model uncertainties. The velocity command, $v_{c}(t)$, is 


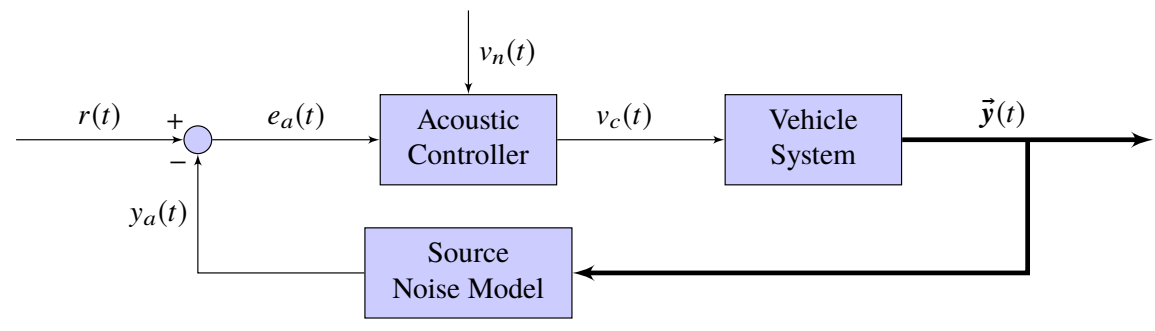

Fig. 1 Simplified block diagram of acoustic controller, vehicle system, and source noise model. Vector signals are shown thicker than scalar signals.

provided to the path following controller, along with the desired point on the path, $\overrightarrow{\boldsymbol{s}}_{\boldsymbol{d}}(t)$. The path following controller ensures the commanded velocity does not drop below the stall speed of the GL-10 and passes it through to the flight dynamics controller, along with the commanded roll and pitch rates, $\overrightarrow{\boldsymbol{c}}(t)$. The flight dynamics controller creates a set of control surface commands, $\overrightarrow{\boldsymbol{u}}(t)$, and these are sent to the vehicle dynamics model.

The vehicle dynamics model embodies the differential equations governing the flight dynamics of the vehicle, with aerodynamic coefficients determined from wind tunnel testing. Equations of motion governing the GL-10 flight dynamics were derived by the Dynamics Systems and Control Branch at the NASA Langley Research Center based on the work of Murphy et al. [8]. The vehicle dynamics output, $\vec{y}(t)$, is comprised of the vehicle attitude, roll, pitch, and yaw rates; the vehicle position, velocity, and acceleration; and the individual propeller speeds, thrusts, and torques of all GL-10 propellers. The latter three are used in the source noise model, as will be shown later, and the other signals in the vehicle dynamics output are subsequently fed back into the path following and flight dynamics control-laws.
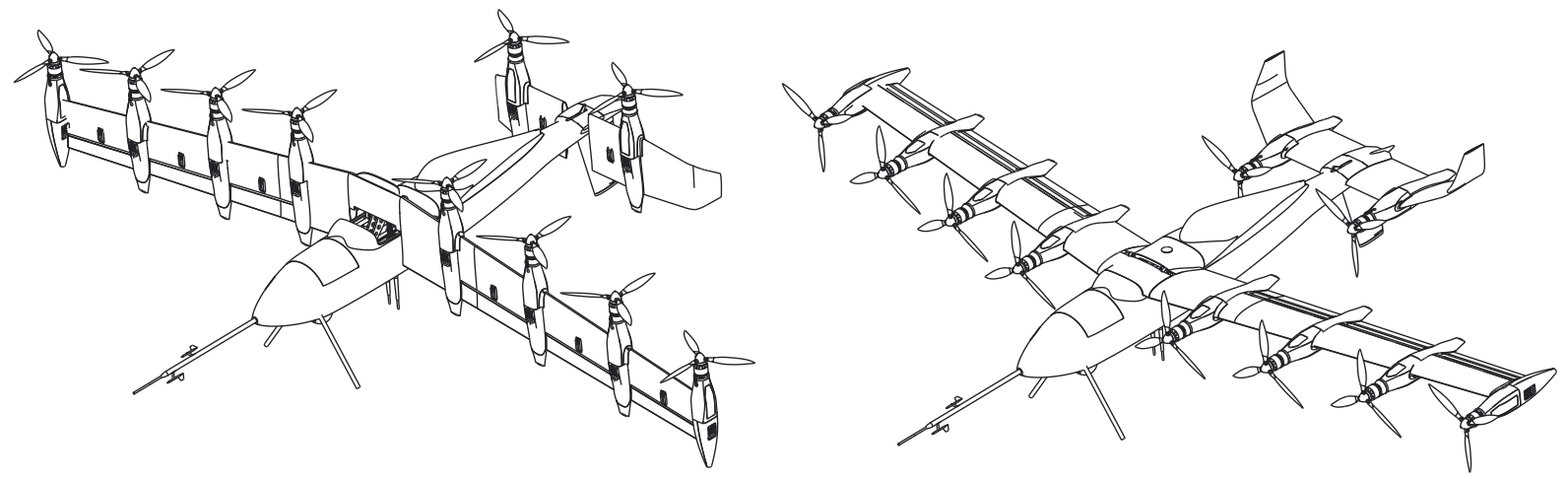

Fig. 2 GL-10 prototype in both hover and cruise flight configurations.

\section{B. Energy Consumption Model}

The fourth component in the vehicle system, shown in Fig. 3, is an energy consumption model that can be used to evaluate the operating efficiency of the vehicle with and without acoustic control. Energy consumed could be a performance parameter in the future, and implementation of the energy consumption model allows for analysis of this during a mission.

The model is based on data collected during an experiment in the anechoic chamber within the Structural Acoustics Loads and Transmission (SALT) facility at the NASA Langley Research Center. This test was conducted to characterize the sound generated by a 3-bladed, 16.0x8.0" folding propeller, driven by a $360 \mathrm{Kv}$ motor, at various motor speeds. This motor and propeller combination is presently used on the GL-10. Results from the experiment are presented in Table 1.

A regression analysis was performed on motor speed and power, shown in

Table 1 Power measured at motor speeds.

\begin{tabular}{cc}
\hline \hline Motor Speed (rpm) & Power $(W)$ \\
\hline 2006 & 35 \\
2500 & 61 \\
3005 & 100 \\
3510 & 157 \\
4006 & 233 \\
4510 & 333 \\
5011 & 465 \\
5519 & 625 \\
6004 & 820 \\
\hline \hline
\end{tabular}




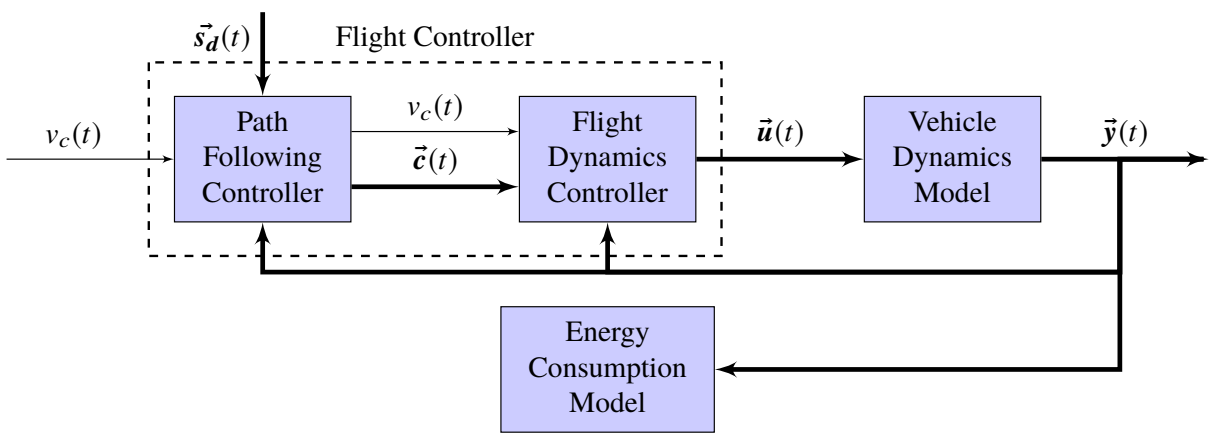

Fig. 3 Block diagram of the vehicle system.

Table 1, to create a model of the power consumption:

$$
P_{m}=4.867 \times 10^{-5} \dot{\theta}^{2}-0.1991 \dot{\theta}+249.0
$$

in which $P_{m}$ is the estimated power consumed by the motor, and $\dot{\theta}$ is the motor speed. During the GL-10 simulations, the power consumption for each motor at each time step was computed using Eq. (1). The wing and tail power consumptions were integrated with respect to time over the duration of the simulation and summed to calculate the total energy used during the mission.

$$
E=\sum_{n=1}^{8} \int_{0}^{t_{f}} P_{\text {Wing }_{n}} d t+\sum_{n=1}^{2} \int_{0}^{t_{f}} P_{\text {Tail }_{n}} d t
$$

Equation (2) is the proposed energy consumption model, in which $E$ is the energy used in Joules. The total energy used will serve as the metric for comparison of electrical efficiency between operating states. Accounting for the wing and tail motors separately is important as the flight dynamics controller sends separate thrust commands to the wing and tail motors.

\section{Source Noise Model}

In this feasibility study, a sound metric and a source noise model are necessary to incorporate an acoustic target into the flight control system. This section will provide a brief background of the sound metric, the source noise model, and the model's implementation.

This initial work uses total radiated sound power level, or sound generated by the source, as the acoustic constraint. Although total sound power is not likely to be the constraint ultimately used in future work, its computation is simple and it avoided the complications of estimating sound pressure level at distant observers, as discussed by Pascioni and Rizzi [9].

\section{A. Background}

The source noise model used in this study estimates the total radiated sound power level of the vehicle and is based on classical analytical expressions proposed by Gutin [10], which captures steady loading noise on the propellers. Although limited in complexity, the Gutin equation is a good candidate for a low fidelity model as it has been shown to provide good results for the first few harmonics [11]. Additionally, the Gutin equation is an analytical model that can easily be implemented in simulation.

There are certain limitations to consider when using the Gutin formula. Gutin stated that this model should only be used for stationary noise estimation without forward flight, however, literature suggests that it can be acceptable for low speed forward flight when the vehicle speed is small compared to the speed of sound [12]. Also, thickness noise, quadrupole terms, and other noise sources are ignored. 


\section{B. Implementation}

Sound power for the GL-10 was computed from sound pressure values given by the Gutin model. Notation adopted by both Gutin [10] and Deming [13] will be used for the sound pressure and power equations and coefficients. First, Gutin gives the root-mean-square (RMS) pressure, $p_{r m s}$, as:

$$
p_{r m s}=\frac{q \omega_{1}}{2 \sqrt{2} \pi c r}\left|-T \cos \vartheta+Q \frac{n c}{\omega_{1} R^{2}}\right| J_{q n}(k R \sin \vartheta)
$$

in which $q$ is the harmonic index, $\omega_{1}$ is the blade passage frequency, $c$ is the speed of sound, $r$ is the distance from the center of the propeller to the observer, $T$ is the propeller thrust, $\vartheta$ is the directivity angle from the axis of rotation, $Q$ is the torque applied to the propeller, $R$ is the propeller radius, and $J_{q n}(k R \sin \vartheta)$ is the Bessel function of the first kind. Figure 4 shows that the propeller rotates about the $x$-axis at a rate of $\Omega, r$ lies in the $x-y$ plane at an angle $\vartheta$ away from the $x$-axis, and $\theta$ lies in the azimuthal plane, in which positive $\theta$ is in the direction of the propeller rotation. The blade passage frequency, $\omega_{1}$, is:

$$
\omega_{1}=n \Omega
$$

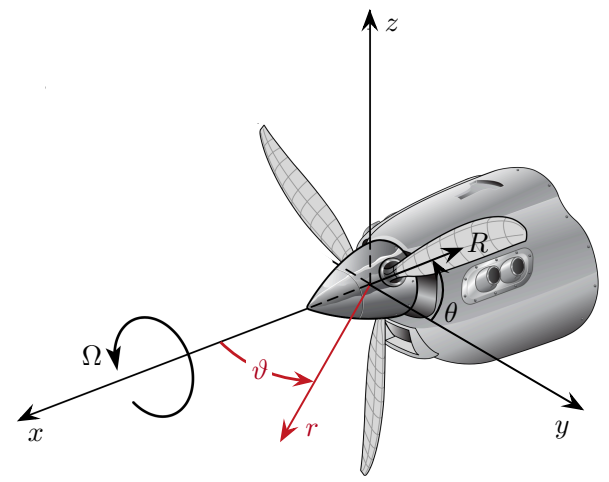

Fig. 4 Gutin model propeller geometry. Figure modified from Zorumski and Weir [14].

in which $n$ is the number of propeller blades. Necessary for the Bessel argument in Eq. (3) is the acoustic wave number, $k$, defined as:

$$
k=\frac{q \omega_{1}}{c}
$$

It is evident from Eq. (3) that loading noise is highly dependent on the rotational rate of the propeller, $\Omega$, thrust, $T$, produced by the propeller, and torque, $Q$, exerted on the propeller. To develop a model for total radiated sound power level, it is necessary to relate sound pressure to sound power:

$$
W=\int_{0}^{\pi} \frac{p_{r m s}^{2}}{\rho c} 2 \pi r^{2} \sin \vartheta d \vartheta
$$

in which $\rho$ is the density of air and $W$ is the sound power of the propeller. Substituting Eq. (3) into Eq. (6) and rearranging yields:

$$
W=\frac{q^{2} \omega_{1}^{2}}{2 \pi \rho c^{3}} \int_{0}^{\pi}\left|-T \cos \vartheta+Q \frac{n c}{\omega_{1} R^{2}}\right|^{2} J_{q n}^{2}(k R \sin \vartheta) \sin \vartheta d \vartheta
$$

As Eq. (7) is a difficult expression to develop a closed-form solution for, the sound power is numerically integrated and updated at every time step during simulation. This results in a model that is a function of propeller speed, thrust, and torque, which are values easily extracted from the GL-10 simulation. The GL-10 has 8 motors mounted to the wings and 2 motors mounted to the horizontal stabilizers, with each motor driving a propeller. Thus, the total sound power is:

$$
W_{\text {Total }}=8 W_{\text {Wing }}+2 W_{\text {Tail }}
$$

in which $W_{\text {Total }}$ is the total sound power, $W_{\text {Wing }}$ is the sound power of a single wing propeller, and $W_{\text {Tail }}$ is the sound power of a single tail propeller. Computing the total sound power level from Eq. (8) results in the following model:

$$
L_{W, \text { Total }}=10 \log _{10}\left(\frac{W_{\text {Total }}}{W_{0}}\right)
$$

in which $L_{W}$ is the total radiated sound power level and $W_{0}$ is the reference power, $10^{-12} \mathrm{~W}$.

\section{Control Approach}

This section describes the control approach used to integrate an acoustic metric into the flight control system. Discussion of the acoustic controller will include a description of the control-law itself, as well as anti-windup functionality that has been implemented. 


\section{A. Proportional-Integral Controller}

A detailed block diagram of the control architecture is presented in Fig. 5. The control architecture proposed for this project tracks an acoustic reference by modifying the velocity command to the path following controller. The path following controller then provides commands to the flight dynamics controller to follow a prescribed path. The acoustic controller uses a Proportional-Integral (PI) controller wrapped around the vehicle system as an outer loop. An acoustic target serves as a reference command, $r(t)$, and is determined a priori based on mission defined acoustical constraints. This reference command is filtered using a second-order low pass filter to produce $r_{a}(t)$, which prevents sharp changes in the command to the acoustic controller.

Noise generated by the vehicle is estimated using the source noise model. Specifically, propeller speed, thrust, and torque of each motor from the system output vector, $\overrightarrow{\boldsymbol{y}}(t)$, are fed into the source noise model to generate a new acoustic estimate, $y_{a}(t)$, based on the current operating state of the vehicle. The acoustic error, $e_{a}(t)$, is calculated by subtracting the noise estimate from the filtered acoustic reference, $r_{a}(t)$, and is defined in Eq. (10):

$$
e_{a}(t)=r_{a}(t)-y_{a}(t)
$$

The acoustic controller updates the velocity command to the path following controller, $v_{c}(t)$, based on the acoustic error signal. The new commanded velocity is defined as:

$$
v_{c}(t)=v_{n}(t)+\Delta v_{a}(t)
$$

in which $v_{n}(t)$ is the nominal velocity command, and $\Delta v_{a}(t)$ is generated using the PI controller presented in Eq. (12), assuming no saturation:

$$
\Delta v_{a}(t)=K_{p} e_{a}(t)+K_{i} \int_{0}^{t} e_{a}(\tau) d \tau
$$

in which $K_{p}$ and $K_{i}$ are tuneable gains of the PI controller. Integral control was added to reduce the steady state error to zero. Derivative control, commonly used in this class of controllers, provided no benefit and was not used in this control-law. Combining Eqs. (11) and (12) produces Eq. (13), defining the relationship between the acoustic error, the nominal velocity command, and the modified velocity command.

$$
v_{c}(t)=v_{n}(t)+\left[K_{p} e_{a}(t)+K_{i} \int_{0}^{t} e_{a}(\tau) d \tau\right]
$$

The updated speed command will increase or decrease the vehicle's velocity through higher or lower motor speeds, thereby increasing or reducing the vehicle noise, $y_{a}(t)$. By analyzing vehicle dynamics and acoustic response during simulations, appropriate gains were tuned to $K_{p}=3 \mathrm{~m} / \mathrm{s} \cdot d B^{-1}$ and $K_{i}=1 \mathrm{~m} / \mathrm{s}^{2} \cdot d B^{-1}$.

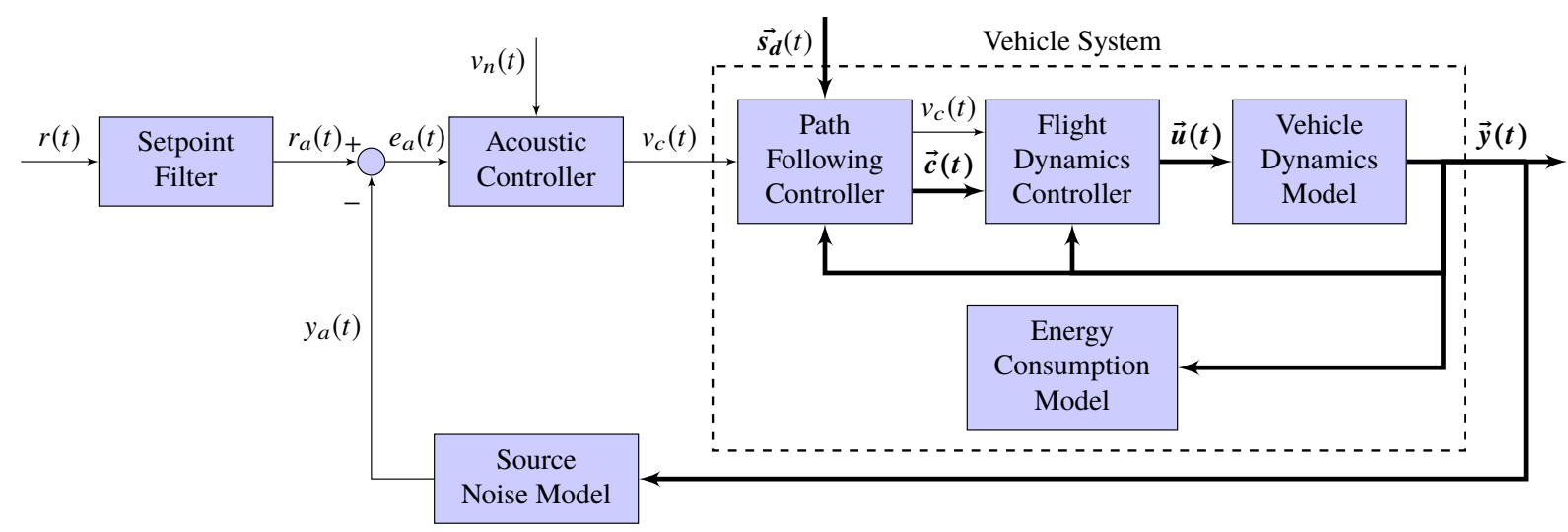

Fig. 5 Detailed block diagram of acoustic feedback controller and vehicle system model. Vector signals are shown thicker than scalar signals.

\section{B. Anti-Windup Logic}

Although a filter was designed to condition the acoustic reference to prevent unrealistic commands to the flight control system, it is still necessary to implement integrator anti-windup logic in the acoustic controller. It is possible 
that the acoustic controller will force the vehicle outside of the flight envelope during a mission as the aircraft operates with time-varying acoustic targets. In this case, error accumulated while the vehicle speed is capped by the flight control system will result in a longer response time once the vehicle returns to a state in which the acoustic controller can function properly. This anti-windup logic will aide in maintaining stable flight throughout the mission and reduce the response time due to integral error once the aircraft returns from an acoustic target outside of its operational capacity.

As shown in Fig. 6, the control signal, $u(t)$, is saturated to create $u_{\text {sat }}(t)$. Saturating the control input additionally protects the flight control system from a high amplitude velocity command in the event that the setpoint filtering is not sufficient. The logic in the saturation block in Fig. 6 is defined as:

$$
u_{\text {sat }}(t)= \begin{cases}M_{\max } & u(t)>M_{\max } \\ u(t) & M_{\max } \geq u(t) \geq M_{\min } \\ M_{\min } & M_{\min }>u(t)\end{cases}
$$

in which $M_{\max }=31 \mathrm{~m} / \mathrm{s}$, just below the the maximum flight speed of the GL-10, and $M_{\min }=19 \mathrm{~m} / \mathrm{s}$, just above the stall speed of the vehicle. The difference between the control signal and the saturated control signal (i.e., the saturation error) is multiplied by a gain, $K_{b}$, and subsequently fed back into the integral path of the PI controller. Incorporating anti-windup into Eq. (13) results in the following control-law:

$$
v_{c}(t)=v_{n}(t)+\left[K_{p} e_{a}(t)+\left(K_{i}+K_{b} u_{s a t}(t)-K_{b} u(t)\right) \int_{0}^{t} e_{a}(\tau) d \tau\right]
$$

The anti-windup gain affects how quickly the integral term is reset to avoid accumulating excessive error. By running simulations and observing how quickly the unsaturated velocity command, $u(t)$, leveled out, the saturation error gain was tuned to $K_{b}=100 s^{-1}$.

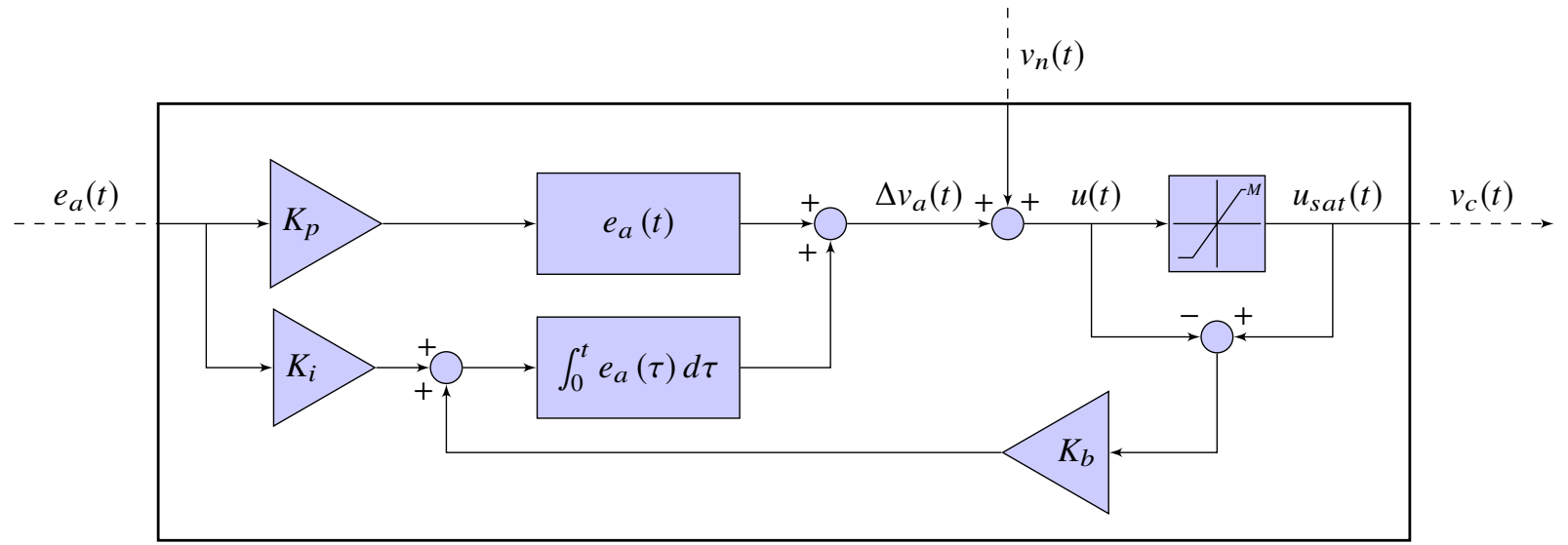

Fig. 6 Detailed block diagram of acoustic controller with anti-windup.

\section{Simulation Results}

Simulations were performed to evaluate the performance of the acoustic controller and the anti-windup logic. This section will begin with discussion of a simulated flight mission operating with and without the acoustic controller enabled. Then, a discussion of data collected to validate the anti-windup logic will follow. Lastly, results of the energy consumption model will be discussed to compare energy usage with and without the acoustic controller enabled.

\section{A. Acoustic Controller Performance}

To evaluate the performance of the acoustic controller, a simulation was performed that encompassed multiple acoustic targets. A set of three acoustic targets were considered - low, medium, and high. These acoustic targets could be appropriate for residential, commercial, and agricultural zones, each of which might have a different sensitivity to a noise source. The low, medium, and high acoustic targets were respectively 121, 125, and $129 \mathrm{~dB}$ (referenced to $W_{0}$ ) sound power level. It is important to stress that the acoustic metric is based on source noise, and not sound pressure at 
an observer. These target sound power levels were arbitrarily chosen to demonstrate the concept in this initial study. For all simulations, the nominal mission trajectory is straight-and-level, unaccelerated flight (SLUF) (i.e., constant velocity).

Figure 7 presents results of a flight simulation of the GL-10 operating under acoustic control. The simulation was started at $t=-20$ seconds to allow the aircraft simulation to reach a trimmed state and SLUF before the acoustic controller was enabled at $t=0$ seconds. The desired altitude was specified as $30.5 \mathrm{~m}$. Once the acoustic controller was enabled, a medium acoustic target was set, followed by a low acoustic target, and ending with a high acoustic target. This demonstrated the concept of a vehicle operating through regions with varying noise constraints.

As shown in Fig. 7(a), the system tracked the acoustic command very well, converging to the value within 5 seconds or less for each acoustic command change. At approximately 46 seconds, the acoustic controller had a $1 \%$ overshoot, which was acceptable for the purposes of this study.

Figure 7(b) presents the velocity and altitude of the aircraft during the simulation. The nominal velocity command, $v_{n}(t)$, was a constant $28 \mathrm{~m} / \mathrm{s}$, indicated by the dashed black line. Once the acoustic controller was enabled, it reduced the commanded velocity from 28 to $\sim 26 \mathrm{~m} / \mathrm{s}$. There is some overshoot in the velocity command, as seen at 0,20 , and 40 seconds, but overshoot is less in the vehicle noise and velocity. The change in velocity command resulted in some error in altitude tracking due to the phugoid mode of the aircraft dynamics. However, the deviation was small enough to be of no concern.

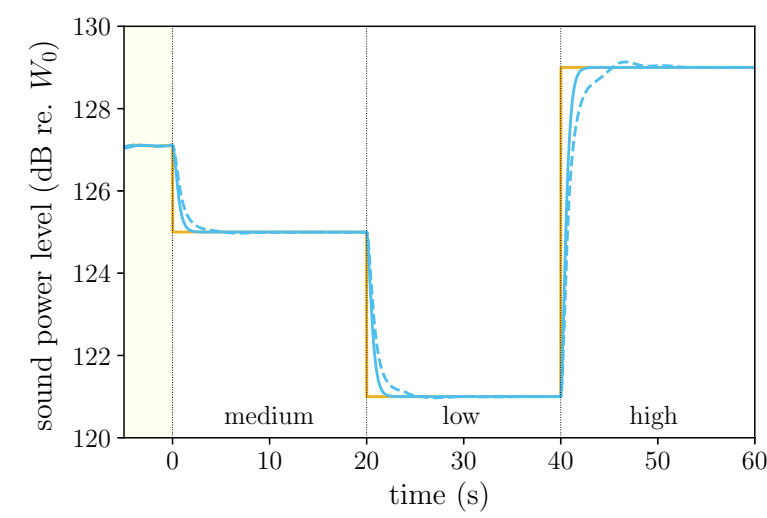

(a) Sound power level vs. time; acoustic target ( - ), command ( - ), and acoustic state ( ---- ).

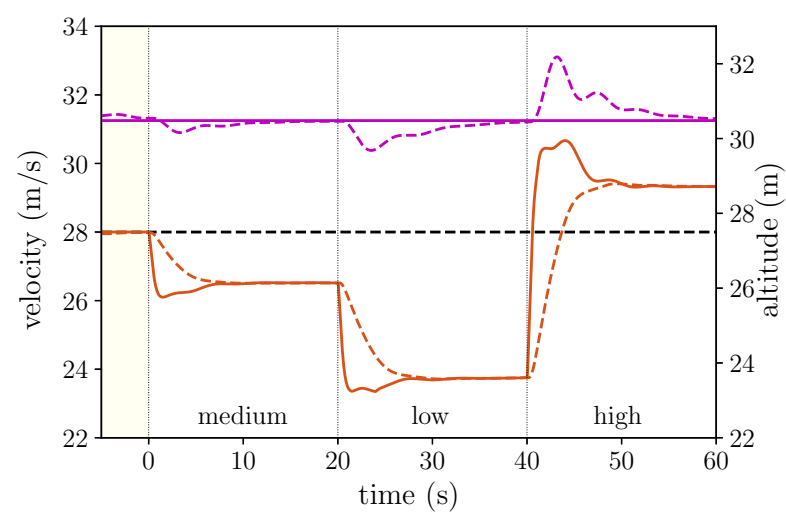

(b) Velocity and altitude vs. time; nominal command ( ---- ), controller command ( - ), and vehicle velocity (---- ); altitude command ( - ) and vehicle altitude ( ---- ).

\section{Fig. 7 Flight simulations with acoustic control enabled.}

\section{B. Anti-Windup Logic Validation}

Two simulations (of the mission described in Sec. V.A), without and with anti-windup enabled, were performed to validate this feature. In the first simulation, an acoustic reference value, larger than the sound power level the vehicle could produce, was sent to the acoustic controller, which commanded a velocity above the saturation limit of $31 \mathrm{~m} / \mathrm{s}$, shown in solid green in Fig. 8(a). This resulted in a steady state error, shown in Fig. 8(b), in which the proportional term (shown in solid light blue) converged to $\sim 10 \mathrm{~m} / \mathrm{s}$ and the integral term (shown in solid red) increased without bounds.

A second flight simulation was performed, and with anti-windup enabled, there are differences in the proportional and integral term behavior. The proportional term initially becomes larger than without anti-windup enabled, due to the initial steady state error, however, the integral term decreases to cancel the proportional term, driving the saturation error to zero. This is illustrated in Fig. 8(a) as the unsaturated velocity command, shown in orange red with stars, converges to the saturated velocity command of $31 \mathrm{~m} / \mathrm{s}$.

\section{Energy Consumption Model}

It was expected that operating under acoustic control would affect the energy consumed, and the following simulations were performed to investigate the trade-offs between acoustically-aware and nominal flight. The first flight simulation was the same as the one shown in Fig. 7 and the second was a nominal SLUF mission without acoustic control.

It was necessary to compare data over equal distances because one simulation resulted in a further distance traveled. 


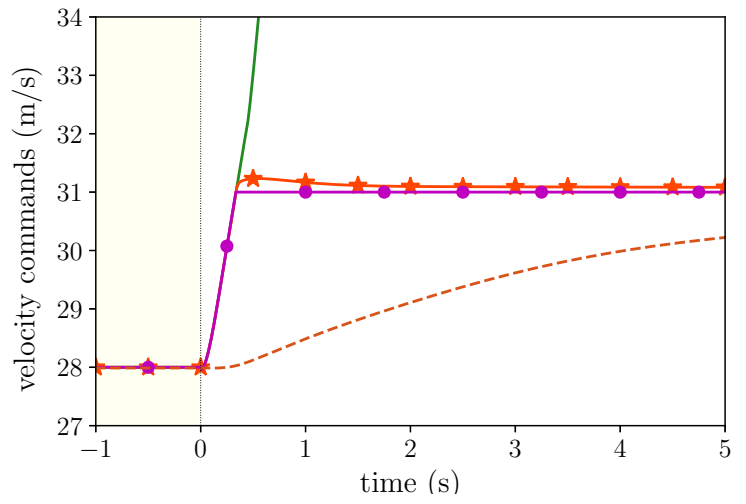

(a) Velocity commands vs. time; unsaturated velocity command without anti-windup enabled ( - ), unsaturated velocity command with anti-windup $(\rightarrow-)$, saturated velocity command ( -- ), and vehicle velocity with anti-windup enabled ( ---- ).

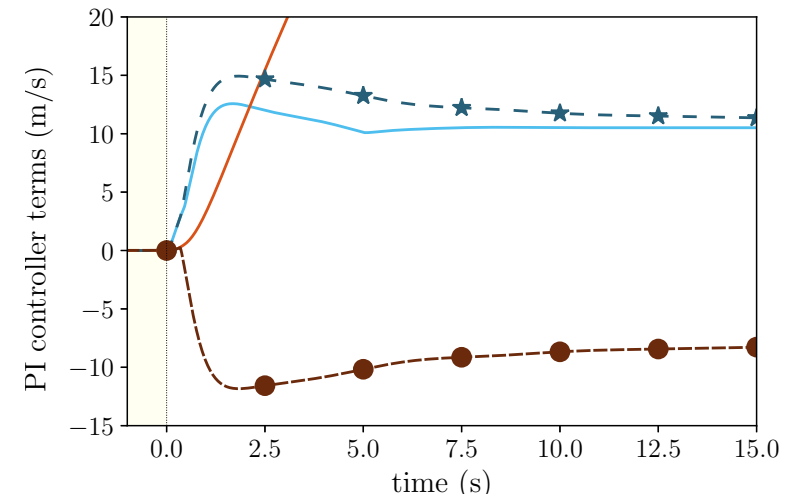

(b) PI controller terms vs. time; proportional term ( - ) and integral term ( - ) without anti-windup logic enabled; proportional term ( - *-) and integral term ( -- ) with anti-windup logic enabled.

Fig. 8 Flight simulations with and without anti-windup engaged.

The limiting case was the simulation with acoustic control enabled, in which the total distance traveled during the 60 second mission was $1589 \mathrm{~m}$. For this case, over the $1589 \mathrm{~m}$ traveled, the GL-10 motors used $1156 \mathrm{~kJ}$ of energy. With acoustic control off, the GL-10 consumed $1235 \mathrm{~kJ}$ to travel the same $1589 \mathrm{~m}$ as the simulation with acoustic control enabled. Therefore, with the acoustic controller enabled, the GL-10 took 5.7\% more time to reach a target distance of $1589 \mathrm{~m}$, while consuming $6.4 \%$ less energy. This result may be intuitive, as motors running at a slower speed consume less energy, but nonetheless, the model provides the capability for energy consumption analysis in future missions in which the results may not be so apparent.

\section{Conclusions and Future Work}

The purpose of this work was to create an acoustic controller and to demonstrate its use for modifying the commanded velocity of an aircraft based on time-varying acoustic targets. A PI acoustic controller was designed and implemented. Anti-windup functionality was implemented to prevent excessive error accumulation in the integrator term in the presence of command saturation. An energy consumption model was developed to allow for analysis of energy used during missions. Simulations were performed to investigate the efficacy of the proposed acoustic controller and anti-windup logic. Simulation results show that the acoustic controller tracks a total radiated sound power target well, adjusting vehicle speed to converge to the target level within 7 to 10 seconds. Anti-windup logic was able to prevent the controller from accumulating excess error. The energy consumption model showed that, for this specific mission, the GL-10 used less energy under acoustic control. This work resulted in a flight control system that provided automatic management of the sound generated during flight, enabling sound to be another performance metric for consideration during mission planning.

Future work will include changing the acoustic targets to spatial constraints instead of temporal targets and incorporating a time lead feature to anticipate upcoming acoustic zones. Trajectories based on these acoustic zones will be optimized a priori, and a more sophisticated acoustic model will be implemented to enable an acoustic metric based on noise at a ground observer. 


\section{Acknowledgments}

M. B. Galles would like to thank Dr. Kyle Pascioni (of the National Institute of Aerospace) and Dr. Stephen Rizzi from the Aeroacoustics Branch, and additionally Dr. Irene Gregory and Dr. John Cooper from the Dynamic Systems and Control Branch at the NASA Langley Research Center for their feedback, guidance, and support with the GL-10 vehicle model. Additional gratitude is expressed to Dr. Randolph Cabell in the Structural Acoustics Branch for his feedback

and Mr. Robert McSwain from the Aeronautics Systems Engineering Branch for assistance with the GL-10 flight characteristics. Lastly, appreciation is extended to Dr. Nikolas Zawodny for providing power consumption data from experiments conducted in the SALT facility. This work was supported by the Revolutionary Vertical Lift Technology project under the Advanced Air Vehicles Program in the National Aeronautics and Space Administration.

\section{References}

[1] Rothhaar, P. M., Murphy, P. C., Bacon, B. J., Gregory, I. M., Grauer, J. A., Busan, R. C., and Croom, M. A., "NASA Langley Distributed Propulsion VTOL Tilt-Wing Aircraft Testing, Modeling, Simulation, Control, and Flight Test Development," 14th AIAA Aviation Technology, Integration, and Operations Conference, AIAA Aviation Forum, Atlanta, Georgia, 2014. doi:10.2514/6.2014-2999.

[2] Fredericks, W. J., McSwain, R. G., Beaton, B. F., and Klassman, D. W., "Greased Lightning (GL-10) Flight Testing Campaign," NASA-TM-2017-219643, NASA Langley Research Center, Hampton, Virginia, 2017.

[3] Cichella, V., Xargay, E., Dobrokhodov, V., Kaminer, I., Pascoal, A. M., and Hovankimyan, N., "Geometric 3D Path-Following Control for a Fixed-Wing UAV on SO(3)," AIAA Guidance, Navigation and Control Conference, AIAA SciTech Forum, Reston, Virginia, 2011.

[4] Xargay, E. M., "Time-Critical Cooperative Path-Following Control of Multiple Unmanned Aerial Vehicles," Ph.D. thesis, University of Illinois at Urbana-Champaign, 2013.

[5] Hovakimyan, N., and Cao, C., $\mathcal{L}_{1}$ Adaptive Control Theory Guaranteed Robustness with Fast Adaptation, Advances in Design and Control, Vol. DC21, Society for Industrial and Applied Mathematics, Philadelphia, 2010.

[6] Hovakimyan, N., Cao, C., Kharisov, E., Xargay, E., and Gregory, I. M., “ $\mathcal{L}_{1}$ Adaptive Control for Safety-Critical Systems,” IEEE Control Systems, Vol. 31, No. 5, 2011, pp. 54-104. doi:10.1109/MCS.2011.941961.

[7] Xargay, E., Hovakimyan, N., Dobrokhodov, V., Kaminer, I., Cao, C., and Gregory, I. M., " $\mathcal{L}_{1}$ Adaptive Control in Flight," Advances in Intelligent and Autonomous Aerospace Systems, edited by J. Valasek, Progress in Astronautics and Aeronautics, AIAA, Reston, Virginia, 2012, pp. 129-172. doi:10.2514/5.9781600868962.0129.0172.

[8] Murphy, P. C., Klein, V., and Frink, N. T., "Nonlinear Unsteady Aerodynamic Modeling Using Wind-Tunnel and Computational Data," Journal of Aircraft, Vol. 54, No. 2, 2017, pp. 659-683. doi:10.2514/1.C033881.

[9] Pascioni, K. A., and Rizzi, S. A., "Tonal Noise Prediction of a Distributed Propulsion Unmanned Aerial Vehicle," 24th AIAA/CEAS Aeroacoustics Conference, AIAA Aviation Forum, Atlanta, Georgia, 2018.

[10] Gutin, L. Ya., “On the Sound Field of a Rotating Propeller,” NACA-TM-1195, NACA, Washington, DC, 1948.

[11] Deming, A. F., "Propeller Rotation Noise Due to Torque and Thrust," The Journal of the Acoustical Society of America, Vol. 12, No. 1, 1940, pp. 173-182. doi:10.1121/1.1916089.

[12] Theodorsen, T., and Regier, A. A., “The Problem of Noise Reduction with Reference to Light Airplanes,” NACA-TN-1145, NACA, Washington, DC, 1946.

[13] Deming, A. F., "Propeller Rotation Noise Due to Torque and Thrust," NACA-TN-747, NACA, Langley Field, VA, 1940.

[14] Zorumski, W. E., and Weir, D. S., "Aircraft Noise Prediction Program Theoretical Manual: Propeller Aerodynamics and Noise," NASA-TM-83199-PT-3, NASA Langley Research Center, Hampton, Virginia, 1986. 